\title{
The role of sleep in changing our minds: A psychologist's discussion of papers on memory reactivation and consolidation in sleep
}

\author{
Rosalind D. Cartwright \\ Sleep Disorder Service and Research Center, Rush University Medical Center, Chicago, Illinois 60612, USA
}

\begin{abstract}
The group of papers on memory reactivation and consolidation during sleep included in this volume represents cutting edge work in both animals and humans. They support that the two types of sleep serve different necessary functions. The role of slow wave sleep (SWS) is reactivation of the hippocampal-neocortical circuits activated during a waking learning period, while REM sleep is responsible for the consolidation of this new learning into long-term memory. These studies provide further insights into mechanisms involved in brain plasticity. Robeiro has demonstrated the upregulation of an immediate-early gene (IEG zif 268) to waking levels, which occurs only in REM and only in connection with new learning. McNaughton and his group have identified electrical indicators that the hippocampus and neocortex are talking to each other by testing the coactivation of hippocampal sharp wave bursts in SWS and shifts from down to up states of activation in the neocortex. In human studies Smith's group reports work on individual differences such as intelligence and presleep alcohol that affect postsleep performance, and Stickgold and collaborators report that a short nap will improve performance if it contains REM sleep. Payne and Nadel suggest that the recall benefit associated with REM sleep may be due to its association with increased cortisol levels. These papers are important not only in their individual contributions but also in revitalizing the work coordinating waking and sleep. This promises to further the understanding of how our unique capacity to learn from experience and modify our behavior takes place.
\end{abstract}

It is fitting that this special issue includes a commentary from Jonathan Winson (2004). He is the acknowledged neuroscientist "father" of this new wave of work looking into the sleeping brain to find a deeper understanding of our unique ability to learn throughout life. As Winson inspired many neuroscientists to continue looking into the dark side of the mind to find the answers of how yesterday's experience is linked to tomorrow's behavior, he, in turn, might nominate Freud as his progenitor. Freud's major work, The Interpretation of Dreams (Freud 1953), besides being responsible for an interminable treatment method, more importantly provided a theoretical framework for a $24-\mathrm{h}$ model of mental behavior. This involved a three-layered representation of continuous streams of thought he called the conscious, preconscious, and unconscious, which shifted in dominance between various states of awareness. This permitted Freud to address such waking anomalies as slips of the tongue and motivated forgetting as well as the meaning hidden in the strange adventures taking place in dreams.

Freud knew that to support this model he must develop a deeper understanding of how the brain works. He made a start on this in his Project for a Scientific Psychology, but had to abandon this effort when he recognized that he did not have the appropriate investigative tools needed to go further. Almost a hundred years later, we have many more of these methods in hand: the various brain imaging techniques, the ability to implant and move about arrays of depth electrodes to record minute activity in single cells, and the more sophisticated data analysis techniques that have informed the work reported in this group of papers. By using these tools, many neuroscientists are now ad-

E-mail rcartwri@rush.edu; fax (312) 942-8961.

Article and publication are at http://www.learnmem.org/cgi/doi/10.1101/ Im.75104. vancing the work to fill in more of Winson's picture of how the off-line sleeping brain contributes activity necessary for waking behavior change. Putting these findings together into a more comprehensive model is an enormous task, and one that needs to be addressed on many levels, at both macro and micro levels, with both rigorous science and thoughtful speculation. At this time the work on this problem has been pursued much more vigorously on the animal level than in humans.

\section{Review of the articles}

The articles appearing here represent a good sampling of this work. They were presented at a recent meeting on memory reactivation and consolidation during sleep. The studies are diverse in many ways. For example, there is the study by Smith and Lapp (1991), which capitalizes on an experiment in nature, the sleep of undergraduate students during periods of high and low loads of "brain work," and a rat study from McNaughton's laboratory (Battaglia et al. 2004) focusing on the temporal relation between some EEG features of slow wave sleep (SWS) in the hippocampus and in the neocortex. These and other studies reported here illustrate the range and remarkable progress in zeroing in on explanatory mechanisms of how both SWS and REM contribute to moving new waking experience into long-term memory. Despite the differences among these approaches, the studies share considerable concurrence in the findings.

So, why does the human work lag so far behind? The evidence that sleep has a positive effect on retention of newly learned verbal material, over and above an equal amount of waking time, dates back to the early 1920s (Jenkins and Dallenbach 1924). Even the idea that the neocortex and hippocampus are reciprocally involved during learning, with activation moving in and out of these areas in waking, and that the same circuits are 
reactivated during sleep is not new. The data supporting that fact that reactivation promotes memory consolidation was reviewed and laid out 20 yr ago in the introductory chapter of Winson's book: Brain and Psyche (1985).

There are several explanations for the relatively slow progress of work of this kind at the human level between the early studies and the present. One is a method problem, that is how sleep was studied. Following the discovery of REM, studies of sleep effects on human learning and memory relied on the classification system for reducing EEG sleep data into the five stages as defined by Rechtschaffen and Kales (1968). These were broadly grouped into REM and non-REM (NREM), or active sleep and SWS, distinguished by which wave forms were dominant in each 30 -sec sleep epoch. This is a rather gross system that has been supplemented by more refined methods, such as spectral analysis, the degree of power at various wave frequencies, and parsing REM sleep into its tonic and phasic components. As recording of human sleep is limited to the surface of the brain, new variables are now being investigated such as hormone levels and their relation to sleep stages and to the quality of dreams. Methods of studying the sleep of animals are becoming even more detailed, with electrical activity of single cells recorded from multiple sites subjected to sophisticated methods of testing their correlation, or coactivation, between the hippocampus and neocortex. Also studies of immediate early gene expression during REM are suggesting that their upregulation is responsible for synaptic changes that consolidate memories. All of this adds up to animal studies that can get a closer look at the brain in action, and "better measures equal better understanding."

A second problem delaying progress in the human work was some uncertainty about what learning tasks were most likely to show a benefit from a sleep period. Many early studies of learning and retention compared performance on very different types of tasks following equal time intervals when REM sleep was present to when it was not. (Empson and Clarke 1970; Barker 1972; Chernick 1972; Grieser et al. 1972; Cartwright 1974; Cartwright et al. 1975). The assumption was that since the brain state of REM is highly active while it is also protected from the interference of new afferent input, it is more likely, than are periods of waking or SWS, to support further processing of new information. These studies were followed by a good deal of skepticism, partly due to their mixed results and to the failure to replicate those showing positive findings. Crossword puzzles, where there is only one correct answer, showed no benefit from REM, but materials involving some affective salience did (Cartwright 1974). For example, a standard emotional story stem was completed in a markedly different manner following a sleep interval containing REM than it was after an equal waking interval. Story completions following waking were rather stereotyped with "happy ending" conclusions, while following REM were more idiosyncratic. This is similar to Stickgold et al.'s (1999) recent report that REM sleep favors "weak primes."

The emphasis on REM actually proved to be another stumbling block to progress in revealing the mechanisms behind the sleep benefit for human learning. Perhaps REM was not the right target. What about the rest of sleep? How does newly learned material, or some uncompleted task, get through SWS to become reactivated in REM? Here, Stickgold et al. (2000) took up the question of whether a direct image representing a new experience could be retrieved from sleep onset awakenings. Do traces of presleep learning cross the wake/sleep barrier, in what form, and how often? Previous psychological studies of incorporation of waking experimental stimuli into REM and NREM mentation reports yielded little evidence. At best were findings of an increase in "symbolic" representations of some emotion arousing experience (Cartwright et al. 1969; Breger et al. 1971). However, few studies investigated whether the sleep onset period included evidence of direct imagery (Vogel et al. 1966). Stickgold (2003) reports that sleep onset awakenings do contain some hypnogogic images of two different video games involving visual and motor components. The work his group reports here looks at the effect of SWS in consolidating, and REM sleep on enhancing, the postsleep performance of newly learned perceptual and motor tasks. He reports that this "two separate sleep functions" finding holds even for short naps with and without REM sleep, a finding previously reported by Barker as quoted in Night Life (1977). When short sleep periods include some REM time, they are followed by enhanced performance not present following those without REM. Stickgold (Kuriyama et al. 2004) added that this improvement also depends on the timing of sleep in relation to the original training period. This confirms Smith's (2003) work showing that not all REM sleep is equal. There are "REM windows," specific times when REM sleep leads to improvement in postsleep performance. This warns that it is not only what sleep is sampled but when to look for an effect.

As more precise measurements have been developed and more fine-grained analyses of sleep and their relation to learning and memory have been undertaken, new variables have emerged as important. The density of the REM eye movements is one such example. The REM sleep of the students in Smith's study (Smith et al. 1991) demonstrated an increase in the total number of eye movements in their sleep close to the examination period without there being an increase in either total REM time or the percentage of REM to total sleep. Perhaps the effectiveness of naps with REM reported by Stickgold (Mednick et al. 2003) will also show that it is those with high eye movement density that are associated with performance enhancement.

To the list of the problems that may be implicated in the slow progress of psychological studies into understanding the "sleep connection" in human learning-the how, what, and when-we must add another: in whom? This relates to the importance of both trait and state variables in the learner. These are highlighted in Smith's report (Smith and Smith 2003) that intelligence helps and alcohol harms postsleep performance. In general it is difficult to carry out these studies in humans, who sleep at such inconvenient times and for such long hours. Studies of human learning and memory were not only few but controversial, until automated monitoring equipment was developed that could be programmed to awaken subjects in their natural home environment after a preset number of eye movements or time into REM to record reports of the prior mental activity or to perform some task. (Mamalak and Hobson 1989; Lloyd and Cartwright 1995). This left most of the progress to come from studies of more accommodating animals. Here there has been a good deal of progress. For example, Dr. Robeiro's work (Robeiro et al. 2002) which also supports that SWS and REM play distinctive but complementary roles in learning goes further than is now possible in human studies, exploring the structural change following exposure to new information. This, he finds is accomplished via the up-regulation of an early-immediate gene to waking levels during REM sleep in the hippocampal-cortical circuit. And McNaughton's group (Battaglia et al. 2004) have also zeroed in on how the neocortex and hippocampus talk to each other in SWS to coordinate the memory traces stored temporarily in the hippocampus by reactivation and to consolidate them into long-term memory. By using arrays of implanted electrodes, they examine the temporal relation of hippocampal sharp wave bursts to the shift from down to up activation states in the neocortex. They suggest that this sequence reflects how a neocortical memory trace is being reprocessed based on information in the hippocampus.

Working at the surface in human studies, Payne and Nadel (2004) report on how they approach the answer to how the two 
major sleep types work to support learning and memory. These investigators chunk sleep into early night, which is predominantly SWS, and late night, when REM predominates, and then examine the types of learning favored by each of these over equal waking intervals. They find that sleep early in the night favors episodic memory; late night, procedural learning. They reason that the last REM of the night, which shows the maximum density of eye movements, may indicate that this period is most responsible for memory consolidation. They argue that the positive postsleep effect on learning may in fact not be due to sleep but to the cortisol level, which increases markedly across the night, with a small increase associated with each REM period, and which reaches its highest level at the end of the night. Perhaps the bizarre, effect-laden dream content characteristic of the last REM episode is due to the heightened cortisol level.

\section{Discussion}

And what about dreams? That's what all the fuss was about back in the early 1950s when REM was primarily of interest because of the distinctive mental content associated with it. That is where both Freud and Winson put their money. To paraphrase Winson (1985): Dreams are the window through which we can observe basic personality structure most clearly. Here we are able to see those issues that motivate, or get in the way of, our making the best use of our special capacity to learn and to change behavior. Winson (1985) suggests that not only does this REM/dream association offer the best opportunity to understand the stable underlying strategies that guide behavior but these can best be observed in the continuity of dream themes across the night. This sequence of dreams reveals the way new experience is being related to older memories. This view of dreams was strongly resisted both by psychologists who rejected Freud for being "unscientific" and by neuroscientists who pointed out that the eye movements of REM are a close parallel to the Pontine-GenicalateOcular Motor (PGO) spikes recorded in the brain stem of the sleeping cat. Since these emanate from the "unthinking" pons, the content of dreams could not be more than accidental. This argument proved to be a major deterrent to further serious work on dreams. Although the public and a small number of investigators were not deterred, laboratory-based studies on the content of dreams slowed almost to a complete stop. The study of continuity of dream themes does present methodological difficulties because of the need to change the subject's state from sleep to waking in order to retrieve a verbal report. This introduces a potential confound. If continuity from dream to dream is demonstrated, can it be claimed that this is inherent or was it introduced during the awakening allowing recall of previous reports?

With the basic work on the role of sleep in learning and memory establishing that all sleep is involved in actively representing, connecting, and relocating new information in the brain, the way is open to again take up the investigation of the psyche. The question is whether dreams, too, contribute to enhancing waking behavior. Do they illustrate the linkages between novel elements in new experience being related to older memory fragments (Paller and Voss 2004) so that we may make more flexible responses to external circumstances and initiate more creative solutions to life's problems, and do they help us "keep our cool" by expressing negative affect safely in dream fantasy? Were Freud and Winson right in pointing to dreams as holding information key to understanding our basic behavior strategies? The studies reported here have made this question respectable again by providing the evidence that the reactivation of neural circuits during SWS involve multiple memory systems representing different aspects related to new experience. If these are then transferred to the neocortex during hippocampal bursts, they can not be expected, McNaughton (McNaughton et al. 2003) reminds us, to consist of one-toone representation of the waking experience. Dream stories are not direct mirrors of waking thoughts. They appear to be constructed from a patchwork of both the new and older memory traces. This leaves some interesting questions open for further work. Of all the new experience of a day, what determines the selection of what is saved for further processing? What is the role of affect in this process? If sleep is so good for learning and memory, why are the dreams themselves so hard to recall? And why is it that we can be reminded of a dream later in the day? Where has it been? Since consolidation of new learning takes time, when should we look for the effects of dreaming on waking life? Do repetitive dreams represent memory nodes critical in understanding the stable behavior strategies Winson (Winson 1985) suggests are the stuff of dreams? At the least, these articles support that sleep is a necessary piece in completing the puzzle of how we humans work. Psychologists should take courage and pick up the task they abandoned of building and testing a new 24-h model of the mind behind our characteristic behavior.

\section{References}

Barker, R. 1977. The effects of REM sleep on the retention of a visual task. In Night life: Explorations in dreaming (ed. R. Cartwright), pp. 94-95. Prentice Hall, Englewood Cliffs, NJ.

Battaglia, F. Sutherland, G., and McNaughton, B. 2004. Hippocampal sharp wave bursts coincide with neocortical "up-state" transitions. Learn. Mem. (this issue).

Breger, L., Hunter, I., and Lane, R. 1971. The effect of stress on dreams. Psychology Issues 7: (monograph).

Cartwright, R. 1974. Problem solving: Waking and dreaming. J. Abnorm. Psychol. 83: 451-455.

Cartwright, R., Bernick, N., Borowitz, G., and Kling, A. 1969. The effects of an erotic movie on the sleep and dreams of young men. Arch. Gen. Psychiatry 20: 262-271.

Cartwright, R., Lloyd, S., Butters, E., Weiner, L., McCarthy, L., and Hancock, J. 1975. Effects of REM time on what is recalled. Psychophysiology 12: 561-568.

Chernick, D. 1972. Effect of REM sleep deprivation on learning and recall by humans. Perception Motor Skills 34: 283-294.

Empson, J.A. and Clarke, P.R. 1970. Rapid eye movements and remembering. Nature 227: 287-288.

Freud, S. 1953. The interpretation of dreams. Hogarth Press, London.

Grieser, C., Greenberg, R., and Harrison, R. 1972. The adaptive function of sleep: The differential effects of sleep and dreaming on recall. $J$. Abnorm. Psychol. 80: 280-286.

Jenkins, J. and Dallenbach, K. 1924. Obliviscence during sleep and waking. Am. J. Psychol. 35: 605-612.

Kuriyama, K., Stickgold, R., and Walker, M. 2004. Sleep-dependent learning and motor skill complexity. Learn. Mem. (this issue).

Lloyd, S. and Cartwright, R. 1995. The collection of home and laboratory dreams by means of an instrumental response technique. Dreaming 5: 63-73.

Mamelak, A. and Hobson, A. 1989. Dream bizarreness as the cognitive correlate of altered neuronal behavior in REM sleep. J. Cogn. Neurosci. 1: 201-220.

McNaughton, B., Barnes, C., Battaglia, F., Bower, M., Cowan, S., Ekstrom, A., Hoffman, K., Houston, F., Karten, Y., Lipa, P., et al. 2003. Off-line reprocessing of recent memory and its role in memory consolidation: A progress report. In: Sleep and brain plasticity (eds. P. Maquet et al.), pp. 225-246. Oxford University Press, Oxford, UK.

Mednick, S., Nakayama, K., and Stickgold, R. 2003. The restorative effect of naps on perceptual deterioration. Nature Neurosciences 5: $677-681$.

Paller, K. A. and Voss, J. 2004. Memory reactivation and consolidation during sleep. Learn. Mem. (this issue).

Payne, J. and Nadel, L. 2004. Sleep dreams and memory consolidation: The role of stress hormone cortisol. Learn. Mem. (this issue).

Rechtschaffen, A. and Kales, A. 1968. A manual for standardized terminology, techniques and scoring system for sleep stages of human subjects. U.S. Government Printing Office, Washington, DC.

\section{Learning \& Memory}


Ribeiro, S., Mello, C., Velho, T., Gardner, T., Jarvis, E., and Paulides, C. 2002. Induction of hippocampal long-term potentiation during waking leads to increased extra-hippocampal zif 268 expression during ensuing rapid-eye movement sleep. J. Neurosci. 22: 10914-10923.

Smith, C. 2003. The REM sleep window and memory processing. In Sleep and brain plasticity (eds. P. Maquet et al.), pp. 117-133. Oxford University Press, Oxford, UK.

Smith, C. and Lapp, L. 1991. Increases in the number of REMs and REM density following an intensive learning period. Sleep 14: $325-330$.

Smith, C. and Smith, D. 2003. Ingestion of alcohol just prior to sleep onset impairs memory for procedural but not declarative tasks. Sleep 26: $185-191$.
Stickgold, R. 2003. Memory, cognition and dreams. In Sleep and brain plasticity (eds. P. Maquet et al.), pp. 17-39. Oxford University Press, Oxford, UK.

Stickgold, R., Scott, L., Rittenhouse, C., and Hobson, A.J. 1999. Sleep induced changes in associative memory. J. Cog. Neuroscience 11: $182-193$.

Stickgold, R., Malia, A., Maguire, D., Roddenberry, D., and O'Connor, M. 2000. Replaying the game: Hypnagogic images in normals and amnesics. Science 290: 350-353.

Vogel, G., Foulkes, D., and Trosman, H. 1966. Ego functions and dreaming during sleep onset. Arch. Gen. Psychiatry 14: 238-248.

Winson, J. 1985. Brain and psyche: The biology of the unconscious. Anchor Press, New York.

. 2004. To sleep, perchance to dream. Learn. Mem. (this issue). 


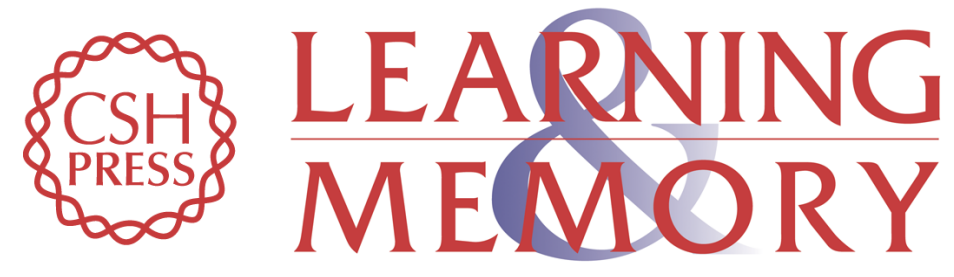

\section{The role of sleep in changing our minds: A psychologist's discussion of papers on memory reactivation and consolidation in sleep}

Rosalind D. Cartwright

Learn. Mem. 2004, 11:

Access the most recent version at doi:10.1101//m.75104

References This article cites 13 articles, 2 of which can be accessed free at: http://learnmem.cshlp.org/content/11/6/660.full.html\#ref-list-1

License

Email Alerting

Receive free email alerts when new articles cite this article - sign up in the box at the Service top right corner of the article or click here. 